\title{
Variable Speed Limits: Strategies to Improve Safety and Traffic Parameters for a Bottleneck
}

\author{
M. Z. Hasanpour \\ Transportation Research \\ Institute, Road, Housing \& \\ Urban Development Research \\ Center Tehran, Iran
}

\author{
M. R. Ahadi \\ Transportation Research \\ Institute, Road, Housing \& \\ Urban Development Research \\ Center Tehran, Iran
}

\author{
A. S. Moghadam \\ Transportation Planning \\ Shomal University \\ Iran
}

\author{
G. A. Behzadi \\ Transportation Research \\ Institute, Road, Housing \& \\ Urban Development Research \\ Center Tehran, Iran
}

\begin{abstract}
The primary purpose of the speed limit system is to enforce reasonable and safe speed. To reduce secondary problems such as accidents and queuing, Variable Speed Limits (VSL) has been suggested. In this paper VSL is used to better safety and traffic parameters. Traffic parameters including speed, queue length and stopping time have been pondering. For VLS, an optimization decision tree algorithm with the function of microscopic simulation was used. The results in case of sub saturated, saturated and supersaturated at a bottleneck are examined and compared with the Allaby logic tree. The results show that the proposed decision tree shows an improved performance in terms of safety and comfort along the highway. The VSL pilot project is part of the Road Safety Improvement Program included in Iran's road safety action plan that is in the research process in the BHRC Research Institute, Road and Housing \& Urban Development Research that is planned for next 10-year Transportation safety view Plan.
\end{abstract}

Keywords-variable speed limits; decision trees; safety, transportation; highway; traffic

\section{INTRODUCTION}

Worldwide, an estimated 1.2 million people are killed in road crashes each year and as many as 50 million are injured [1]. Current strategies recognize that speed management should be central and have refocused attention on road and vehicle design and related protective features. The "blame the victim" culture is superseded by blaming the traffic system which throws illegal speed on the shared responsibility and accountability for a Safe System [2]. International research has shown that the Variable Speed Limits (VSL) system is a successful traffic engineering application used to manage traffic conditions during congestion, incidents, road building, and inclement weather [3]. VSL could be implemented in appropriate areas to disappear off the potential for driver error, excessive speeds, and the velocity differential between cars and to enhance safety [4]. Also, very different studies have been performed that quantify the predicted benefits of implementing comprehensive VSL control strategies on state highways. In [5, 6] authors used microscopic simulation to examine the impacts of VSL responses to real-time traffic safety standards. In [5], it was found that that, for highly congested areas, VSL provided a reduction in crash potential of $25 \%$, but increased travel time.
In contrast, in [6] it was found that VSL provided a large reduction in crash potential during low traffic (higher speed) conditions, but had little impact for peak period conditions. Authors in [6], likewise comprise a uniform decrease in travel time for the Low traffic conditions using VSL, although the relative change in travel time from the non-VSL is controlling the condition to VSL controlling condition was very depressed.

In [7] , authors evaluated the impact of VSL in an urban freeway in Toronto, Canada. The study was conducted using the microscopic simulator PARAMICS combined with a categorical crash model developed in [5]. The VSL algorithm used was based on a logic tree that uses threshold values for a traffic flow, occupancy, and average travel speed. Each VSL sign was associated to an adjacent loop detector, and each sign operated individually. The outcomes of the simulation indicated that the implementation of VSL signs could significantly improve safety, however the authors concluded that the use of VSL signs increased the travel time for all traffic scenarios considered. In [8], authors applied a method to explore the parallels between ramp metering and applying VSL upstream of a potential bottleneck or high volume merging situation. The metanet second order macroscopic model was modified to allow the VSLs to be integrated.The study showed that when applied upstream, the VSL can act similarly to ramp metering where the current is brought back on the mainstream sooner than along the incline. The traffic arriving at the bottleneck is temporarily cut down and the system delays propagation of the congestion. Four scenarios were evaluated: no-control, VSL control, ramp metering, and integrated instruction. The VSL case decreased total time spent in the system (TTS) by $15.3 \%$, and when VSLs and ramp metering are used in conjunction the TTS were reduced by as much as $19.5 \%$. The survey concluded that traffic flow and mental ability can be improved through VSL use by boiling down the capacity drop at bottlenecks. Nevertheless, if the VSL is applied at under-critical conditions without the potential for bottleneck mitigation, mean speed is turned down and flow efficiency is diminished. Regarding these results and the limitations of empirical studies, the expected overall benefit of implementing VSLs is still unclear [9]. 
The purpose of this survey concentrates on fine tuning the algorithm of VSLs algorithms for Improve Safety and Traffic Parameters. Traffic parameters includes speed changes, queue length and time period. To optimize the VSL algorithm, a decision tree algorithm was used. In the following, the VSL performance in the three following conditions, including the sub saturated, the saturated and supersaturated at a bottleneck examined and compared with the results of an Allaby logic tree.

\section{DESCRIPTION OF STUDY NETWORK}

To set a proper authorized speed and examine its effect on safety and terrific parameters, a specific Part of the haraz road in Iran was selected. A system of traffic, pavement and visibility sensors will be set up to observe conditions and provide operations staff with recommended speeds. Iran's road weather or traffic road conditions are shifting rapidly, particularly in high altitude mountain passes; operations staff will continuously monitor the conditions information and set the digital variable speed signs accordingly. Overhead message signs will let travelers know when they're entering a variable speed zone, and warn drivers to be aware of changing weather and traffic conditions. Variable speed limits will be enforced by the police.

The reason of choosing this part of the road is the change in the number of lanes and the bottleneck at this location. The study path is approximately 4 kilometers long. Information and data required for the research are obtained from cameras installed in the studied area. The set-up is shown in Figure 1. Traffic volume, vehicle types, density per 20 seconds and rate of 10 vehicles per one minute are the first obtained information. The vehicles are considered as 3 types of personal vehicles, vans and buses. Authorized speed was 100 kilometers per hour in the two- lane section and 60 kilometers per hour in the one-lane section and the peak hour of traffic was 3:00 pm till 6:00 pm.

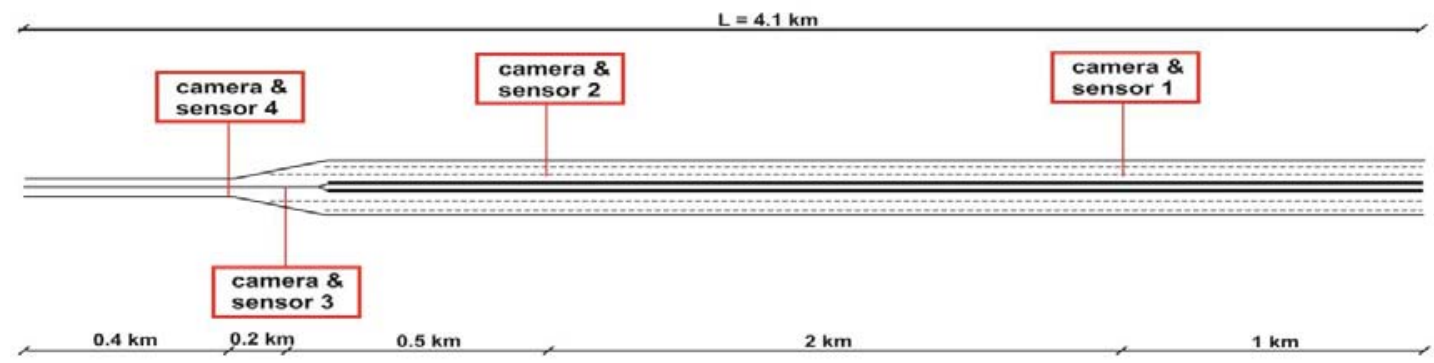

Fig. 1. Exposure intervals cameras

\section{SIMULATION DEVELOPMENT: BASE MODEL}

In [7], authors used simulation software PARAMICS, to simulate VSL which speed was determined based on the decision tree. Figure 2 shows a cross-sectional region of the study and Figure 3 summarizes the logic used in the algorithm. Each VSL sign connects to the adjacent sensor. For low volume (less than 1,600 vehicles per hour), "occupancy", has been practiced as a constituent of the variables for reducing speed. For high volume (more than 1600 vehicles per hour) "occupancy" is not counted. Finally, according to the average speed, the speed is determined and displayed. Simulation results showed that VSL singes can improve safety by reducing speed, but increase the travel time for all considered traffic conditions. Figure 4 shows, speed changes before and after implementation of VSL at study region [10]. In this method the highway is divided into specific sections, which each section has a sensor and VSL sign and acts as a unit. Then by using of statistical information of traffic of each department (average current rate, and the rate of occupancy) thresholds and decision parameter sets and a tree structure for reaching conclusions about traffic speed limits in the different conditions forms.

\section{A. New Model}

For the analysis and simulation of the network, AIMSUN simulation software is used. AIMSUN is one of the best microscopic simulation software and the ability to compound static and dynamic approaches in a single environment is its unique characteristic. Before the simulation, AIMSUN should be calibrated to create a network same as the real one to observe the changes after creating a new management system. To do this, at first, path practical density was estimated according to following equations and finally compared with the AIMSUN output.

$$
\begin{aligned}
& \mathrm{C}=\mathrm{C}_{\mathrm{J}} N F_{\mathrm{W}} \mathrm{F}_{\mathrm{HV}} \mathrm{F}_{\mathrm{P}} \\
& \mathrm{SF}_{\mathrm{i}}=2800 *\left(\frac{\mathrm{V}}{\mathrm{C}}\right) \mathrm{f}_{\mathrm{W}} \mathrm{f}_{\mathrm{HV}} \mathrm{f}_{\mathrm{d}}
\end{aligned}
$$

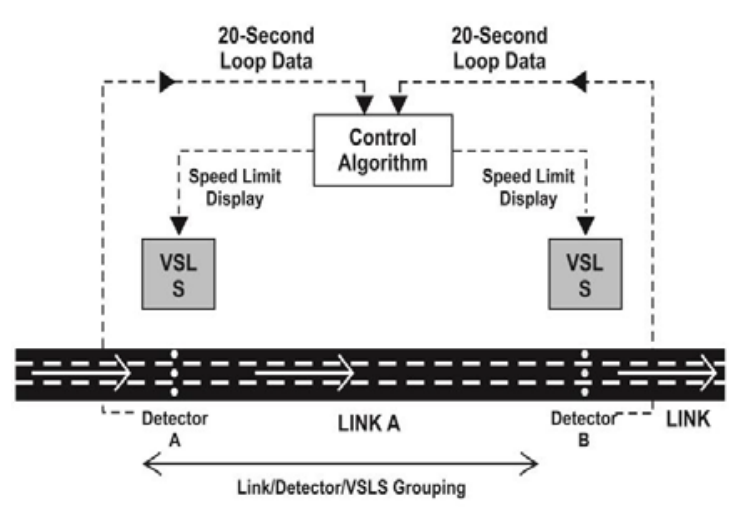

Fig. 2. The plan of implementation of VSL signs and sensors 


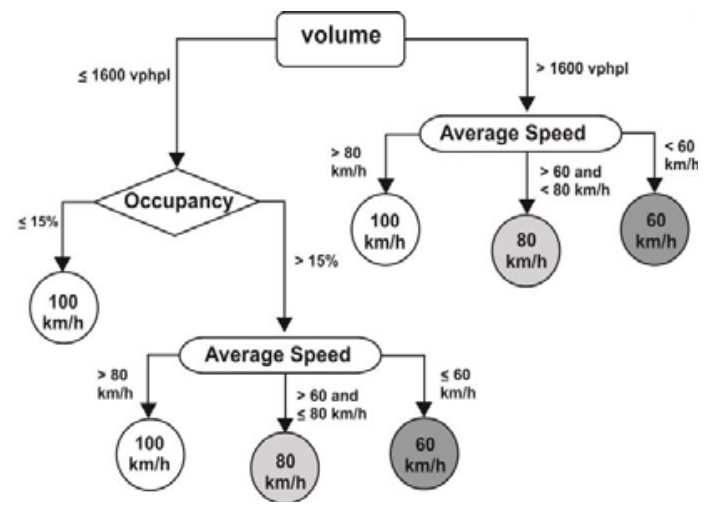

Fig. 3. Decision tree to determine the new speed sent from VSL

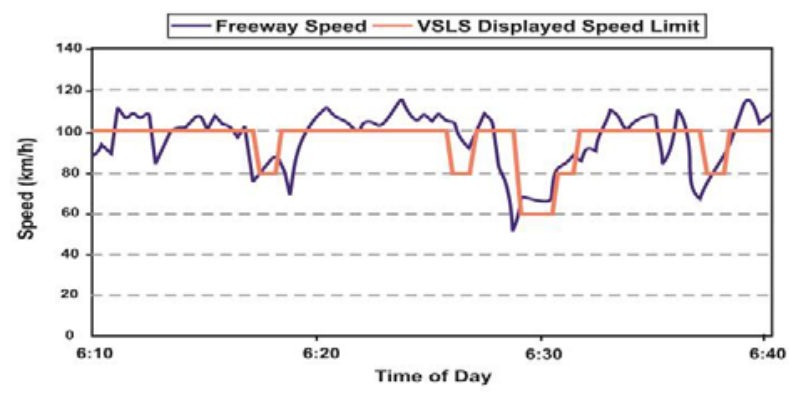

Fig. 4. Speed changes after VSL implementation

The studied network was drawn in AIMSUN software and the primary simulation was performed after calculating the practical capacity of a path. Ultimately, the main turnout was achieved from AIMSUN to calibrate the desired network; the answers indicated that volume, speed and travel time obtained from studying the region, were almost equal with the simulation results that indicated that the path is correctly calibrated in AIMSUN. Rules and the simulation results are shown in Table I. Regarding to gathered information, the practical capacity and the average speed of the studied region was 1900 vehicles per hour and 70 kilometers per hour, respectively.

TABLE I. RESULTS OF EMPIRICAL AND PRACTICAL OUTPUT

\begin{tabular}{|c|c|c|}
\hline Practical results & Simulation results & Variable \\
\hline Volume & $1959 \mathrm{veh} / \mathrm{hr}$ & $1830 \mathrm{veh} / \mathrm{hr}$ \\
\hline Average speed & $70 \mathrm{~km} / \mathrm{hr}$ & $68 \mathrm{~km} / \mathrm{hr}$ \\
\hline Travel time & $47.5 \mathrm{Sec} / \mathrm{km}$ & $53 \mathrm{Sec} / \mathrm{km}$ \\
\hline
\end{tabular}

\section{THE OPTIMAL DECISION TREES}

WEKA software is used for designing the decision tree. WEKA provides a slightly complete collection of the latest machine learning algorithms and data pre-processing tools for data mining. This software is designed so that different algorithms can be examined on new data collections and includes the algorithms for all standard problems of data mining such as regression, classification, clustering, mining the association rules, choosing the features and predicting their class based on primary data. The WEKA software package contains the execution of the latest general version of C4.5 decision tree learning or the J48 and decision trees are created automatically through adjusting defined parameters and are displayed as an ASCII statement [11]. Three grades were designed for data:

- E class; which is for speed ranges or same behavior in Los E.

- D class; which is for speed ranges or same behavior in Los D

- B class; which is for speed ranges or same behavior in Los B.

WEKA software needs training data to forecast the track of instruction of the main data and construct the decision trees. For this purpose, the data obtained from the first week of observation were used as the training data. After each data class was placed on the original data, re-analysis was performed and the ultimate decision tree in WEKA was achieved. Two different input groups were used: (1) mean speed, speed variance and volume (2) mean speed, speed variance, density and volume. The decision tree outputs based on group (1) showed less validity whereas the outputs based on group (2) had higher validity in terms of classification and accuracy. High validity of the tree is mainly attributed to the density variable. The constructed decision tree and the statistics are depicted in Figure 5 and Table 2, respectively.

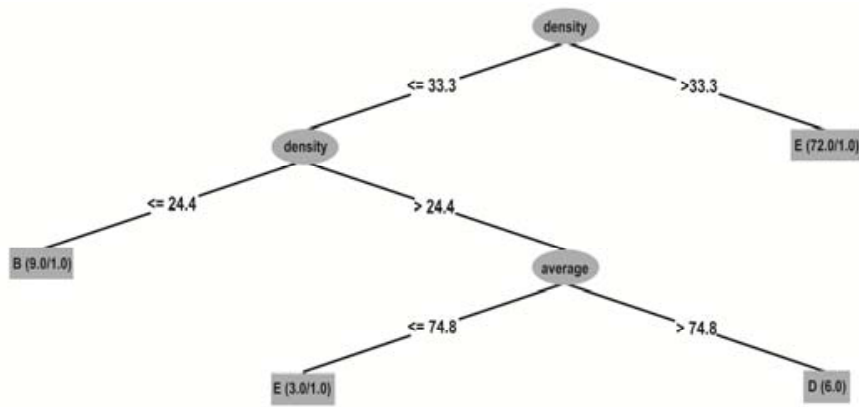

Fig. 5. Decision tree design by WEKA software for: mean speed, speed variance, density and volume

TABLE II. THE RESULTS OF THE DECISION TREE IN DIFFERENT MODES

\begin{tabular}{|c|c|c|c|}
\hline & & $\begin{array}{c}\text { Group (1): mean } \\
\text { speed, speed variance } \\
\text { and volume }\end{array}$ & $\begin{array}{c}\text { Group (2): mean speed, } \\
\text { speed variance, density } \\
\text { and volume }\end{array}$ \\
\hline \multicolumn{2}{|c|}{$\begin{array}{c}\text { Correctly Classified } \\
\text { Instances }\end{array}$} & $86.6 \%$ & $91.1 \%$ \\
\hline \multicolumn{2}{|c|}{$\begin{array}{c}\text { Incorrectly Classified } \\
\text { Instances }\end{array}$} & $13.3 \%$ & $8.8 \%$ \\
\hline \multicolumn{2}{|c|}{ Kappa statistic } & 0.7 & 0.83 \\
\hline \multirow[t]{3}{*}{ TP Rate } & $\mathrm{B}$ & 0.615 & 0.875 \\
\hline & $\mathrm{D}$ & 0.538 & 0.667 \\
\hline & $\mathrm{E}$ & 0.984 & 0.945 \\
\hline \multicolumn{2}{|c|}{ F-Measure } & 0.865 & 0.91 \\
\hline
\end{tabular}

\section{VSL DESIGN NETWORK}

To investigate the created path in three density conditions of sub saturation, saturation, super-saturation, a simulation volume of 1300 to 2700 vehicles per hour is applied to network and the simulation is performed for two conditions (without control and controlled by the decision tree). To implement the decision tree and utilize variable speeds in AIMSUN, three parameters should be fixed. At the beginning, the sensors were placed on simulation regarding to the site of cameras which is 
shown in Figure 6. Then, two parameters of tree constructing were created by producing different policies and triggers and were applied on sensors of the primary tree. Triggers are responsible for the activation and non-activation time. The insurance was based on speed change in this simulation and three types of 60, 80 and 100 speed was produced according to the number of modifications to cope during the itinerary. Selecting 60, 80, 100 authorized speeds is because of the fact that the selected speed should be more eminent than the speed change threshold (50 and 70) so that sensors are able to change over from one active branch to non-active branch and the speed changes are brought near. Eventually, through correct parameter setting, five simulation repetitions were performed for each volume. Results are shown in Table III.

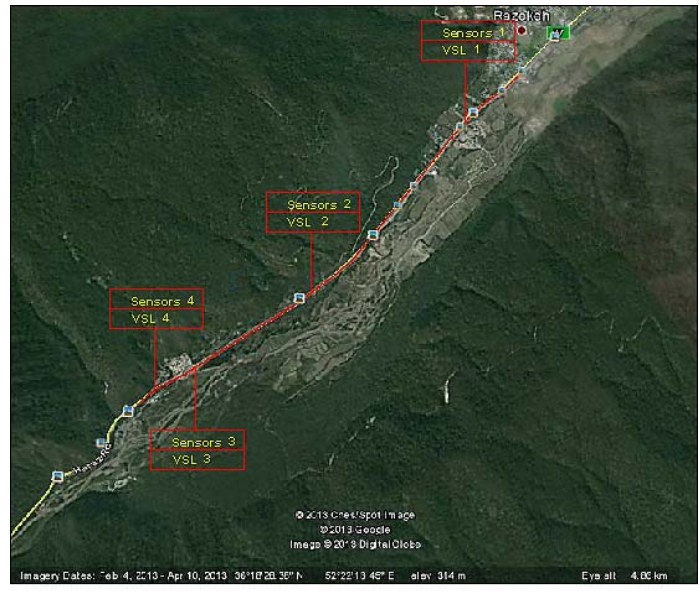

Fig. 6. Positioning of sensors and VSL signs

\section{VSL IMPACT RESULTS}

For analyzing the variation of variables, the proposed decision tree is compared with an Allaby logic tree at three traffic conditions: sub saturated, saturated and supersaturated with and without controlling the VSL signs. Influential variables such as queue length, intermediate speed of travel, the amount of delay, numbers and stops time are examined.

\section{A. VSL Traffic Impacts}

The results regarding queue length are shown in Figure 7. As shown, the VSL helps in reducing the queue length. The biggest reduction happened when the highway reached its capacity. A reduction of about $20 \%$ was reached with the new decision tree, compared to the results of the Allaby logic tree.

\section{B. VSL Safety Impact}

\section{1) Harmonic Speed}

As shown in Figure 8 the graph of average speed at the saturation capacity condition without any VSL is a sharp slope which shows that there is a sudden change in the speed and this an increased possibility for accidents. This improved the application of VSL and it is more improved with the proposed decision tree compared to the Allaby logic tree and a higher average velocity is also achieved.
2) Delay Time, Stop Time, Number of Stop Time

As shown in Figure 9, when the speed limit is controlled by the new decision tree algorithm, the delay, in small volumes increased, but in saturated volume did not interchange. But according to Figures 10 and 11, which shows the vehicles stop time and the number of stops, when the study region has no variable speed limit control vehicles stop time and numbers of vehicle stops is larger. This means that safety is actually increased through the use of VSL as a more smooth movement is achieved.

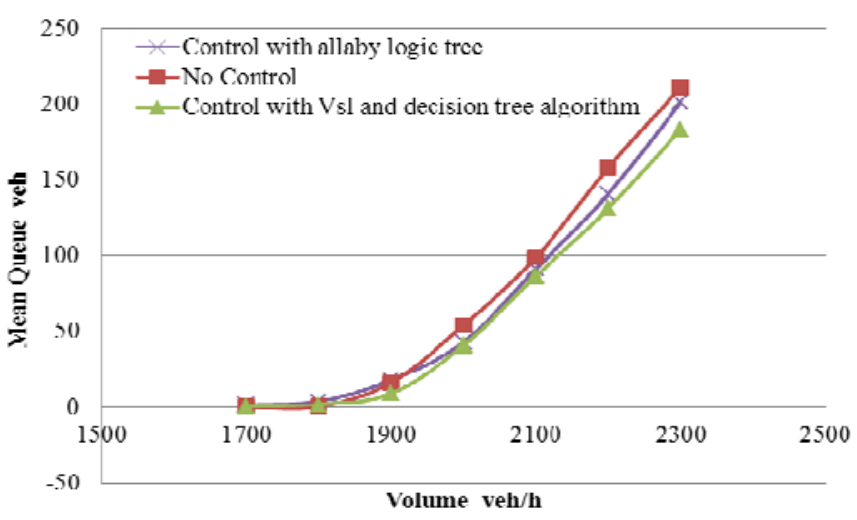

Fig. 7. VSL effect on queue length

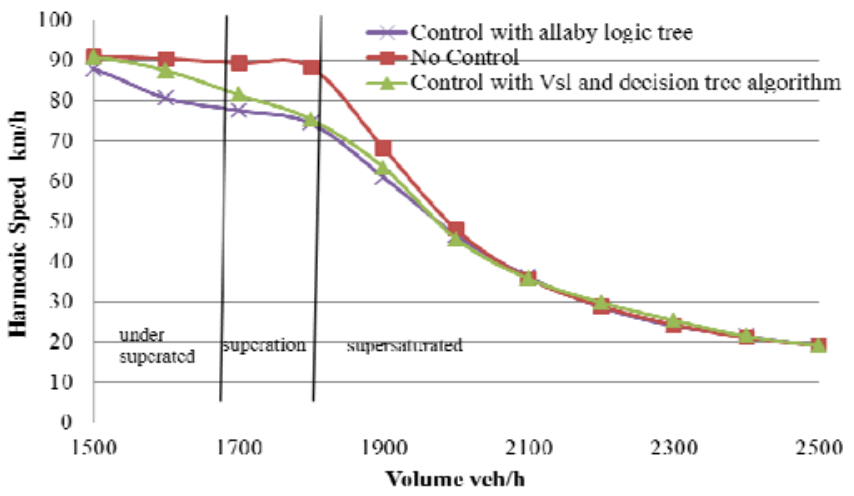

Fig. 8. VSL effect on harmonic speed

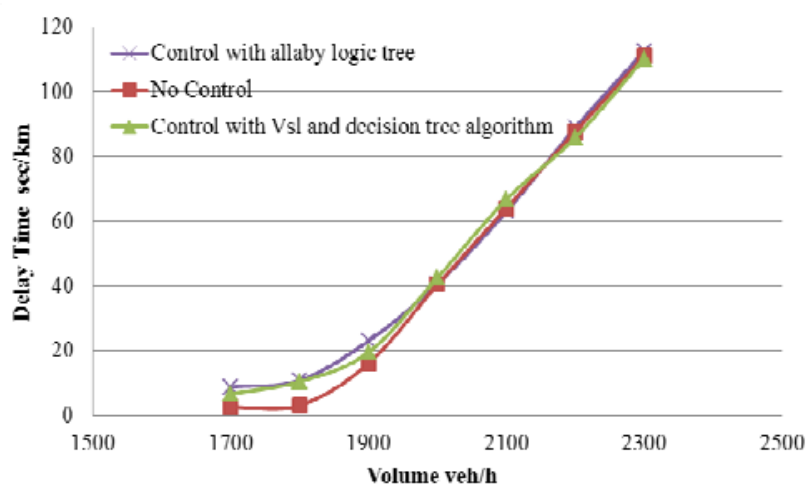

Fig. 9. VSL effect on delay time 


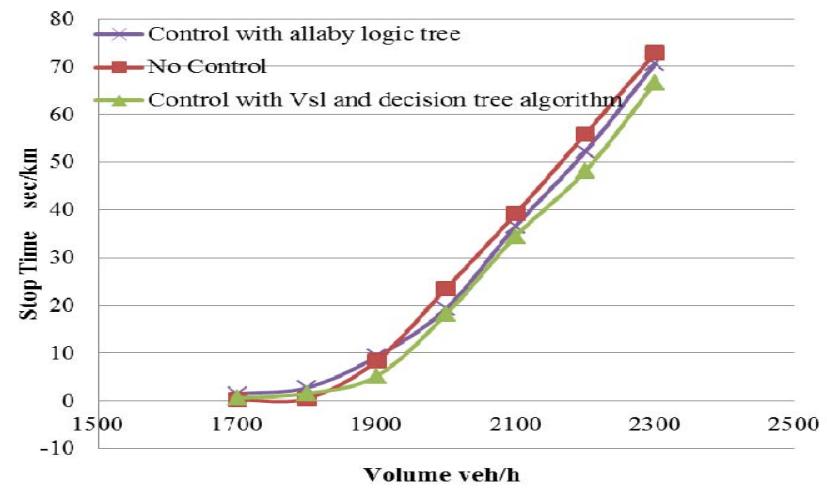

Fig. 10. VSL effect on stop time

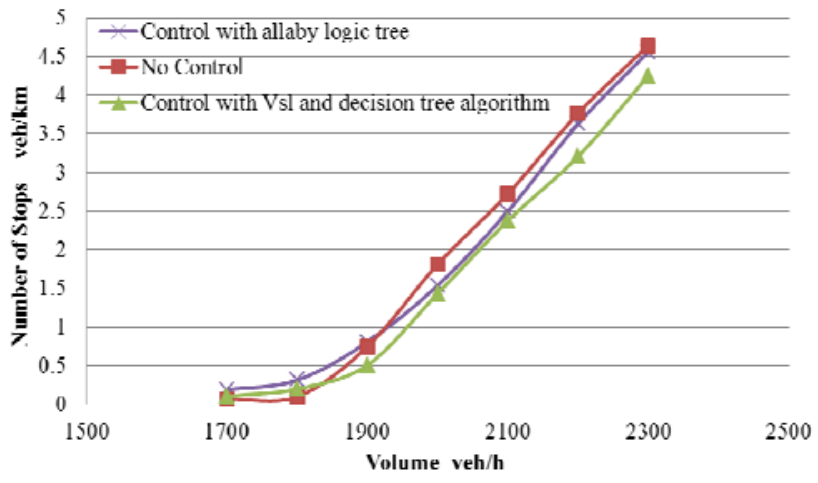

Fig. 11. VSL effect on number of stops

TABLE III. AIMSUN SIMULATION RESULTS

\begin{tabular}{|c|c|c|c|c|c|c|c|c|}
\hline Volume & $\begin{array}{l}\text { Delay Time - } \\
\text { Vehicle }\end{array}$ & $\begin{array}{l}\text { Density - } \\
\text { Vehicle }\end{array}$ & $\begin{array}{c}\text { Flow- } \\
\text { Vehicle }\end{array}$ & $\begin{array}{c}\text { Harmonic } \\
\text { Speed - Vehicle }\end{array}$ & $\begin{array}{l}\text { Standard } \\
\text { Deviation }\end{array}$ & $\begin{array}{l}\text { Mean Queue - } \\
\text { Vehicle }\end{array}$ & $\begin{array}{c}\text { Number of } \\
\text { Stops - Vehicle }\end{array}$ & $\begin{array}{c}\text { Stop Time } \\
\text { Vehicle }\end{array}$ \\
\hline 1300 & 1.518 & 3.78 & 1272.66 & 90.034 & 5.3 & 0 & 0.028 & 0.018 \\
\hline 1400 & 1.68 & 4.09 & 1365.20 & 90.642 & 5.3 & 0 & 0.03 & 0.026 \\
\hline 1500 & 2.002 & 4.42 & 1465.73 & 90.886 & 5.4 & 0.002 & 0.038 & 0.052 \\
\hline 1600 & 2.13 & 4.734 & 1565.2 & 87.602 & 6.5 & 0.028 & 0.048 & 0.108 \\
\hline 1700 & 6.666 & 5.602 & 1653.60 & 81.54 & 7.9 & 0.576 & 0.11 & 0.644 \\
\hline 1800 & 10.446 & 6.432 & 1744.13 & 75.422 & 8.6 & 1.878 & 0.202 & 1.548 \\
\hline 1900 & 19.488 & 8.07 & 1809.19 & 63.456 & 11.5 & 8.986 & 0.51 & 5.2 \\
\hline 2000 & 42.58 & 11.904 & 1827.86 & 45.694 & 13.6 & 40.69 & 1.436 & 18.17 \\
\hline 2100 & 66.772 & 16.276 & 1829.33 & 35.762 & 15.2 & 86.486 & 2.378 & 34.53 \\
\hline 2200 & 85.856 & 20.224 & 1838.66 & 29.874 & 14.8 & 131.11 & 3.21 & 48.14 \\
\hline 2300 & 110.27 & 25.242 & 1837.20 & 25.43 & 13.4 & 183.11 & 4.252 & 66.64 \\
\hline 2400 & 130.31 & 29.95 & 1833.46 & 21.458 & 12.9 & 251.45 & 5.138 & 81.75 \\
\hline 2500 & 150.91 & 34.654 & 1832.93 & 19.128 & 11.9 & 312.46 & 5.648 & 97.62 \\
\hline 2600 & 167.39 & 38.13 & 1831.46 & 17.582 & 11.4 & 355.65 & 6.332 & 110.4 \\
\hline 2700 & 183.63 & 40.85 & 1832.53 & 16.298 & 10.8 & 391.51 & 6.944 & 123.7 \\
\hline
\end{tabular}

\section{CONCLUSION}

VSL is a promising highway traffic management tool for improving traffic safety by offering drivers more realistic speed limits when the driving environments are compromised by traffic congestion, incidents or adverse weather. The present work concentrates on fine tuning the VSL algorithm. Traffic parameters considered includes speed changes, waiting line, time stop. A decision tree algorithm was used for optimization. The VSL performance during a heavy congestion for a bottleneck was considered. Results show that the proposed algorithm decreased the queue length by more than $20 \%$. Further, a smoothing was achieved regarding speed changes and the number of stops and the stop time were reduced resulting to increased safety.

\section{REFERENCES}

[1] World Health Organization, Global Status Report on Road Safety 2013: Supporting a Decade of Action, Geneva, Switzerland, 2013.

[2] T. Bliss, B. Jeanne, Road Safety Management Capacity Reviews and Safe System Projects Guidelines, Working Paper 84203, Washington, DC, World Bank Group, 2013

[3] Federal Highway Administration, Impacts of Technology Advancements on Transportation Management Center Operations Final Report, FHWAHOP-13-008, 2013
[4] Austroads Research Report, Best Practice for Variable Speed Limits: Literature Review, Austroads, Sydney, 2009

[5] C. Lee, B. F. Hellinga, F. Saccomanno, "Assessing safety benefits of variable speed limits”, Transportation Research Record 1897, TRB, National Research Council, Washington, D.C, Vol. 1, No. 1, pp. 183190, 2004

[6] M. Abdel-Aty, J. Dilmore, A. Dhindsa, "Evaluation of variable speed limits for real-time freeway safety improvement”, Accident Analysis \& Prevention,Vol. 38, No. 2, pp. 335-345, 2006

[7] P. Allaby, B. Hellinga, M. Bullock, "Variable Speed Limits: Safety and Operational Impacts of a Candidate Control Strategy for Freeway Applications", IEEE Transactions on Intelligent Transportation Systems, Vol. 8, No. 4, pp. 671-680, 2007

[8] R. Carlson, I. Papamichail, M. Papageorgiou, A. Messmer, "Variable Speed Limits as a Mainline Metering Device for Freeways", Transportation Research Board Annual Meeting, Washington, DC, Paper \#10-1529, 2010

[9] M. Abdel-Aty, R. Cunningham, V. Gayah, L. Hsia, "Dynamic Variable Speed Limit Strategies for Real-Time Crash Risk Reduction on Freeways”, Transportation Research Record No. 2078, Transportation Research Board of the National Academies, Washington, DC, Vol. 1, No. 1, pp. 108-116, 2008

[10] FHWA-SA-12-022, "Guidelines for the use of variable speed limit systems in wet weather”, US Department of Tranportation, 2012

[11] F. Rahmani, F. Ghahari, Weka Software Guide, 2010 